\title{
Philosophiques
}

\section{André Vidricaire et collaborateurs, Figures de la philosophie québécoise après les troubles de 1837, Montréal, Département de philosophie (UQAM), (Collection Recherches et Théories), 1988, vol. II et III.}

\section{Claude Gagnon}

Volume 16, numéro 2, automne 1989

URI : https://id.erudit.org/iderudit/027099ar

DOI : https://doi.org/10.7202/027099ar

Aller au sommaire du numéro

Éditeur(s)

Société de philosophie du Québec

ISSN

0316-2923 (imprimé)

1492-1391 (numérique)

Découvrir la revue

Citer ce compte rendu

Gagnon, C. (1989). Compte rendu de [André Vidricaire et collaborateurs, Figures de la philosophie québécoise après les troubles de 1837, Montréal,

Département de philosophie (UQAM), (Collection Recherches et Théories), 1988, vol. II et III.] Philosophiques, 16(2), 450-455. https://doi.org/10.7202/027099ar d'utilisation que vous pouvez consulter en ligne. 
ANDRÉ VIDRICAIRE ET COLLABORATEURS, Figures de la philosophie québécoise après les troubles de 1837, Montréal, Département de philosophie (UQAM), (Collection Recherches et Théories), 1988, vol. II et III.

$$
\text { par Claude Gagnon }
$$

Entreprendre, ainsi que l'ont fait depuis quelques années les professeurs Malouin et Vidricaire, une étude de la présence de la philosophie dans le vécu 
politique de cette époque était une nécessité. Car trop de nos intellectuels ont préjugé de l'absence d'un vécu philosophique québécois authentique; les travaux publiés par le groupe de recherche Malouin-Vidricaire nous ont déjà dégagé du préjugé et les deux livres dont il est ici question nous permettent un début de jugement proprement scientifique sur le rôle qu'a joué la philosophie de chez-nous après «les troubles de 1837 ".

Nous devons prendre évidemment à la lettre cette périodisation (aprèstroubles-37) référant à notre histoire politique qui qualifie et coiffe la collection co-dirigée par les deux professeurs du département de philosophie de l'UQAM. Cette périodisation permet de ne jamais oublier qu'il s'agit de philosophie située dans un cadre essentiellement politique.

L'introduction d'André Vidricaire ( Procédures en histoire de la philosophie québécoise », vol. II, p. 1 à 31 ) est intéressante à plusieurs points de vue. Concernant le préjugé, Vidricaire montre en un paragraphe-clef l'indéniable présence d'un travail "de nature théorique» (p. 4) chez certains de nos intellectuels d'alors engagés dans divers débats culturels, sociaux ou politiques ; Dessaulles, Aubin, Lanctôt étaient des philosophes. Dans les vestiges écrits de leurs conférences, dans leurs livres et articles doctrinaux ou polémiques, dans leurs correspondances et leurs journaux, ils ont pratiqué la réflexion propre à l'étude des fondements des questions humaines et à la critique radicale des réponses institutionnelles. Ils ont aussi expérimenté leurs recettes et modèles théoriques. Ils ont donc philosophé. C'est précisément ce que l'ensemble des six dossiers ici publiés veut démontrer.

Ainsi orientés autour du pôle politique « 1837 », les professeurs Malouin, Chabot et Vidricaire entourés de leurs collaborateurs (Aubry, Cournoyer, Leclerc et Séfriou) nous font rencontrer les figures philosophiques que furent Dessaulles, Benjamin Pâquet, Médéric Lanctôt, Napoléon Aubin plus quatre sulpiciens représentatifs « de l'idéologie, de la philosophie et du pouvoir clérical au Canada » (A. Séfriou, p. 227).

Le pôle « 1837 » n'est cependant pas le seul horizon théorique des six études; dans son introduction André Vidricaire propose celui de « 1834 » en référence à la publication cette année-là des Paroles d'un croyant par Lamennais. Cette date est bien légitime pour la philosophie de chez-nous puisque Rome condamne le livre et interdit l'enseignement de Lamennais ; « de même que Montalembert et Lacordaire vont se séparer de Lamennais, de même fera le clergé de Saint-Hyacinthe (...) tandis que les jeunes patriotes vont épouser les idées condamnées de Lamennais » écrit Vidricaire qui donne ensuite dans le détail trois exemples positifs (Parent, Dessaulles, Papineau) et un négatif (Sabin Raymond suivant la ligne romaine et Montalembert).

Avec cette périodisation, Vidricaire met en relief cette pierre angulaire qu'est la philosophie lamennaisienne non seulement pour le positionnement des «libéraux de l'Institut Canadien " (p. 19) mais surtout dans le débat sur l'infaillibilité personnelle du pape, débat majeur dans notre société intellectuelle du siècle dernier. Vidricaire dégage de cette conjoncture culturelle un axe 
philosophique important : « l'enjeu est de séparer foi et raison et de départager les sphères de compétence... » (p. 28). Cette séparation disciplinaire est une authentique manifestation d'un syndrome d'une culture savante faisant penser à de multiples situations sociales analogues dans l'histoire universelle de la philosophie; notre philosophie québécoise, encore ici, reprend un thème cher à la pensée du Moyen Âge, celui du rapport entre la Révélation chrétienne et la libre raison.

L'auteur de cette importante introduction termine en relevant des listes d'associations et de périodiques qui nous permettent aujourd'hui, par leur documentation conservée, de circonscrire concrètement les vestiges de ces philosophies dont l'oralité dominante de leur culture (p. 4) et de leur rhétorique (passim) est perdue pour toujours.

La première figure qu'on nous présente est celle de Médéric Lanctôt (1838-1877). Christian Aubry et André Vidricaire rédigent une notice biographique substantielle et tout juste bien épicée de ce bouillant esprit syndical avant la lettre qu'était celui de Lanctôt. Celui-ci, journaliste de carrière, fait ses armes au Pays de l'Institut Canadien (p. 40) et part après pour une croisade qui devrait l'avoir rendu célèbre: promoteur «de la création d'une nation canadienne-française 》 (p. 42), Lanctôt convoque une association générale de tous les corps de métier en 1867 (p. 43). Cinq mille ouvriers répondent! C'est la Constituante de la Grande Association qui regroupera les revendications des principaux ouvriers spécialisés de l'époque et visera le projet d'une société nouvelle. Cette Grande Association, inspirée d'un modèle anglais (p. 52) proposera contre la Confédération l'annexion aux États-Unis.

Ce mouvement annexionniste se fait et s'écrit; les auteurs de la monographie ont choisi de publier l'une des pièces importantes du travail de réflexion de Lanctôt, il s'agit de son essai intitulé $A$ ssociation du capital et $d u$ travail, publié en 1872 (réimpr. p. 164 à 197). La vie de Lanctôt, malgré ses contradictions personnelles, et l'indéniable influence qu'a joué la Grande Association dans la société montréalaise de l'époque nous conduisent à considérer ce texte de 1872 comme l'un des textes importants de notre histoire politique. Ce texte s'inscrit dans la longue tradition moderne des utopies : le principe de Médéric Lanctôt était « l'association du travailleur et du capitaliste ". Il a passé sa vie à essayer de réaliser son rêve humaniste ; estce cependant suffisant pour en faire un philosophe? La lecture de son texte de réflexion sur la question ne révèle aucun travail théorique sur le fondement de la possibilité d'une telle association. Si Lanctôt était philosophe, il a dû faire ce travail et a pu l'écrire ne serait-ce que sommairement. Le dépouillement de l'impressionnante auvre littéraire de Lanctôt, compilée par les aureurs (p. 58 à 158), révélera peut-être au lecteur philosophe cette pierre angulaire sur laquelle ce célèbre orateur a soulevé la conscience d'une bonne partie de sa sociéré.

Marc Chabot nous présente une formule bilbiographique originale pour nous entretenir du libéral catholique Benjamin Pâquet. Plutôt que d'extraire 
une biographie d'une bibliographie, le chercheur a choisi de présenter une bibliographie de Pâquet ordonnée selon son vécu : 240 item composent le grain de la figure philosophique de Pâquet. Chaque notice biographique n'a pas à proprement parler un titre mais bien un appendice littéraire décrivant le contenu de la source sous une forme qui se rapproche du commentaire oral:

« informe l'abbé Bolduc qu'il espère que la question de l'Université sera traitée à la Nouvelle Congrégation du 27 janvier, que les affaires des Sulpiciens n'ont pas progressé depuis sa dernière lettre et l'avise qu'il est décidé de partir de Naples pour l'Orient si la question de l'Université est terminée. 25 janvier 1874 U 36, $n^{\circ} 77$. » (p. 226).

Cette performance réussit à réanimer le personnage en refaisant son œuvre au fil de sa vie. Marc Chabot qui a étudié et pratiqué abondamment les formes épistolaires et journalistiques, nous offre, avec cette biographie animée, une contribution qui répond à la fois aux critères de scientificité des études bibliographiques et à l'exigence du lecteur de biographies qui ne veut pas s'ennuyer. Chabot fait précéder son film bio-bibliographique d'une notice dans laquelle il aborde directement la question de savoir si Benjamin Pâquet peut être considéré comme un philosophe. Sa réponse est claire. «II (Pâquet) est un écrivain de circonstance (...). On peut même avancer qu'il est une figure philosophique mineure, si on utilise comme critère la production philosophique. On trouvera donc de tout dans la bibliographie. Des notes de cours, des sermons, des éloges funèbres, des articles et surtout de nombreux journaux de voyage. Dans l'état actuel de la recherche, d'un point de vue strictement philosophique, son auvre la plus importante demeure Le Libéralisme. » (p. 205; il s'agit des item 104 et 105 de la bio-bibliographie).

Ces cours publics et conférences que Pâquet a donnés à l'université Laval en 1872 permettent, selon l'auteur de la présente étude, de définir Pâquet comme un libéral catholique qui «se différencie d'un libéralisme radical ou athée comme celui d'un Dessaulles» (p. 207). Cette nouvelle recherche sur Pâquet éclaire davantage la polémique du temps autour de l'enseignement des auteurs classiques dans notre culture québécoise très judéo-chrétienne. La figure d'un " libéral catholique », identification composant le titre même de la contribution de Chabot, prend une dimension qui la rend tout à fait concrète en indiquant l'ascendant qu'est Montalembert dans la possibilité d'une telle étiquette idéologique qui fut donc bien réelle pour nous à ce moment-là.

Voilà peut-être pourquoi les éditeurs ont placé tout juste après cette étude de Marc Chabot une bibliographie titrée «Le libéralisme catholique entre 1868-1870». Rédigée par Luc Cournoyer du département d'Histoire à I'UQAM, cette contribution se compose exclusivement d'un repérage bibliographique des articles parus dans le Journal de Québec durant ces deux années qui furent importantes à plusieurs titres pour l'histoire de nos idées puisque 1870 voit la proclamation de l'infaillibilité du pape et la mort de l'un des principaux opposants à cette doctrine, Montalembert. 
Cette question de l'infaillibilité "personnelle " du pape (position ultramontaine de Louis Veuillot, p. 269) a fait l'objet d'un débat théorique tout à fait intéressant chez nos intellectuels d'alors. Dans la seconde partie de son travail, Luc Cournoyer résume un choix d'articles de cette périodisation appliquée au même journal. Il n'y a malheureusement aucun texte de présentation justifiant au non-spécialiste de cette période le choix de ces dates ou lui permettant d'identifier les protagonistes en cause. Qui était l'important " rédacteur» du Journal de Québec qui critiquait Louis Veuillot (11 déc., p. 2) et dénonçait la propagande haineuse de La Gazette des Campagnes? Ce n'est qu'après avoir lu l'ensemble des résumés des articles qu'on peut arriver à identifier certains axes du débat et certains auteurs qu'on aurait bien aimé connaître dès le début.

Le volume II s'achève avec la production d'un texte inédit de Mgr Dupanloup sur le programme d'enseignement de la philosophie et découvert par André Vidricaire aux Archives Nationales de France à Paris (p. 287) ; il s'agit d'une proposition de l'évêque d'Orléans au programme d'une Commission malheureusement non identifiée. Ce texte de Dupanloup mérite tout autant que celui de Lanctôt l'édition que nous offre aujourd'hui l'équipe de recherche en philosophie québécoise de I'UQAM ; ce texte technique, fort précis sur certains points doctrinaux ( «cahier II'»), ne nous apprend malheureusement rien sur les fondements théoriques du libéralisme économique. On pourrait aussi se pencher sur la proposition qui y est faite de supprimer Xénophon, Cicéron, Épictète, tous trois moralistes païens.

En déambulant rue Saint-Denis, lorsque le clocher de l'église SaintJacques miroite une pluie fraîche, on rencontre parfois un vieillard à barbe de patriarche, tenant une canne et portant des bésicles au regard furtif : c'est le fantôme de Napoléon Aubin, philosophe québécois entre tous, autodidacte, journaliste, inventeur-chimiste, complice de Philippe Aubert de Gaspé fils ! Danielle Leclerc présente cette figure de français né en banlieue de Genève en 1812 et arrivant en Amérique à l'âge de seize ans et demi. Ce jeune intellectuel aventurier deviendra chez-nous un journaliste polémiste dont le verbe et l'inépuisable capacité d'écriture étonneront tout le monde jusqu'à sa mort en 1890 à Montréal. Danielle Leclerc propose une biographie détaillée et intelligente du personnage coloré qu'était ce «Démocrite québécois 》 (vol. III, p. 21) ainsi qu'Aubin se définissait lui-même ( «...moi pauvre petit fantasque... je serai Démocrite ", Ibidem). Ses intérêts personnel et professionnel pour les lettres et les arts aussi bien que pour les sciences et les technologies nous permettent de le classer parmi ces esprits universels que furent les humanistes du monde moderne naissant.

Rédacteur-éditeur de plusieurs journaux d'opinion dont le plus célèbre est Le Fantasque, nommé directeur du Canadien en 47 , cofondateur de l'Institut Canadien de Québec en 48, expert en chimie agricole, inventeur d'un gaz d'éclairage qu'il commercialisa lui-même de Saint-Hyacinthe à SanFrancisco, Napoléon Aubin est l'un de nos authentiques philosophes du $\mathrm{XIX}^{\mathrm{e}}$ siècle par sa réflexion théorique et son action sociale et culturelle 
réformatrice. Cet intellectuel d'origine calviniste porté en «terre protestante » à sa mort (p. 45) fut l'un des esprits forts d'un mouvement de Réforme au sens propre du terme qui a orienté depuis le XVI ${ }^{\mathrm{e}}$ siècle l'histoire des idées de l'Occident. Danielle Leclerc complète son étude en nous offrant un choix de courts textes d'Aubin; on y lit une exigence philosophique entre toutes d'Aubin : que les actions remplacent les paroles (p. 73).

Harel Malouin nous présente la suite de son dépouillement des sources concernant Louis-Antoine Dessaulles. Le professeur Malouin travaille Dessaulles depuis quelques années; il annonce un livre (Un Rouge au $19^{\circ}$ siècle: $L . A$. Dessaulles, éditions Saint-Martin). En attendant, il nous révèle deux lettres de Dessaulles à sa fille Caroline comportant des passages passionnants sur le créationnisme et le rapport entre la foi et la raison (p. 209 à 223).

Alain Séfriou termine le vol. III en alignant quatre notices bio-bibliographiques consacrées à autant de sulpiciens: Dominique Graner, Antoine Giband, Louis Colin et Adam Desmazures. L'auteur de la recherche justifie brièvement cette sélection en rappelant que cet ordre religieux a joué un rôle important dans la réalité historique de notre culture (p. 227). Ces hommes cultivés « ont employé leur érudition et leurs talents à essayer de restaurer un ordre social chrétien» (Ibidem). Les textes fournis en complément par Séfriou permettent de rencontrer encore ici l'esprit et la sensibilité de nos ancêtres érudits. Nul doute que ces deux volumes de recherche bibliographique, largement exemplifiée par des textes illustrant toutes les figures des personnages en cause, apporte une connaissance plus positive de l'histoire de notre philosophie.

Département de philosophie

Université du Québec à Trois-Rivières 